Saudi Journal of Medical and Pharmaceutical Sciences

Abbreviated Key Title: Saudi J Med Pharm Sci

ISSN 2413-4929 (Print) |ISSN 2413-4910 (Online)

Scholars Middle East Publishers, Dubai, United Arab Emirates

Journal homepage: https://saudijournals.com/sjmps

Review Article

\title{
Nose to Brain Targeted Drug Therapy: A Review
}

\author{
Dr. M. Vani ${ }^{1 *}$, A. S. Ezhilarasi ${ }^{2}$, Sereena Saju ${ }^{2}$
}

${ }^{1}$ Professor, K.K. College of Pharmacy (affiliated to The Tamil Nadu Dr. M.G.R Medical University), 1/161 Sanakaralinganar Road, KRA Campus Gerugambakkam, Chennai 600 122, India

${ }^{2}$ Pharm.D Interns, K.K. College of Pharmacy (affiliated to The Tamil Nadu Dr. M.G.R Medical University), 1/161 Sanakaralinganar Road, KRA Campus Gerugambakkam, Chennai 600 122, India

DOI: $10.36348 /$ sjmps.2020.v06i11.002

| Received: 12.10.2020 | Accepted: 29.10.2020 | Published: 05.11.2020

*Corresponding author: Dr. M. Vani

\section{Abstract}

Neurodegenerative diseases (NDD) are a major global public health concern and a leading cause of disability-adjusted life-years, moreover, therapeutic options are limited. Brain targeting has always been challenging due to the presence of various physiological barriers, of which one of the most robust barriers is Blood Brain Barrier (BBB). The BBB consists of endothelial cells which are the tight layers surrounded by astrocyte foot processes, and these anatomic features together constitute a significant barrier that hampers the delivery of drugs to the central nervous system (CNS). In the past few decades, the nose-to-brain targeted delivery has received a great deal of attention because of factors like the rapid onset of action, increased bioavailability, manageable surface area and avoids the first-pass metabolism, thus considering it as a non-invasive, convenient and reliable approach that may overcome the challenges associated with nose-to-brain targeted delivery. However, this strategy still possesses significant limitations, which led to the development of nanotechnological approaches like solid lipid nanoparticles, polymeric nanoparticles, nanoemulsions, liposomes and polymeric micelles, thus circumventing these barriers. The present review article highlights a complete understanding of every aspect related to nose-to-brain delivery: mechanism involved in the transport of drugs from noseto-brain via BBB by complex pathways, strategies to overcome the barrier-BBB, recent examples, and applications of nose-to-brain targeted delivery using nanotechnologies for brain gliomas and other neurological disorders.

Keywords: Nose-to-brain, Brain Targeting, Blood Brain Barrier (BBB), Bioavailability, Neurodegenerative disorders (NDD), Glioblastoma (GBM), Nanocarriers.

Copyright ( ) 2020 The Author(s): This is an open-access article distributed under the terms of the Creative Commons Attribution 4.0 International License (CC BY-NC 4.0) which permits unrestricted use, distribution, and reproduction in any medium for non-commercial use provided the original author and source are credited.

\section{INTRODUCTION}

Neurological disorders are a group of disorders that affect the structure or function of the central nervous system (CNS). Alzheimer's disease (AD), dementia, brain gliomas, Parkinson's disorder (PD), Stroke and Transient Ischemic Attack (TIA), Epilepsy and other seizure disorders are some of the brain-related disorders.

A systematic analysis performed for the Global Burden of Disease (GBD) study 2015 stated that Neurological diseases are the world's largest cause of disability-adjusted life years (DALYs), or years of healthy life lost to due to death. The study included neurological disorders such as stroke, $\mathrm{AD}$ and other dementias, PD, epilepsy, multiple sclerosis, migraine, tension-type headache, medication overuse headache, meningitis, tetanus, encephalitis, brain and nervous system cancer. The systemic analysis ranked neurological disorders as the leading cause group of
DALYs in 2015 (250.7 million, comprising $10.2 \%$ of global DALYs) and the second-leading cause group of deaths (9.4 million), comprising $16.8 \%$ of global deaths [1].

The major challenge in the delivery of drugs to the brain is the presence of a physiological barrier that restricts the delivery of drugs to the CNS, namely the Blood-brain barrier (BBB) [2]. Hydrophilic substances, proteins, charged molecules, and peptides are unable to cross the BBB, whereas lipophilic drugs (antidepressants, anxiolytics and many hormones) can easily cross the endothelial cells in the BBB [3]. Despite of tremendous advancement in the treatment of neurological disorders, there is still a need for a novel brain targeted drug delivery system and a non -invasive transport of drug to the brain.

Nose-to-brain drug delivery system is a targeted and a non-invasive approach in which drug is targeted in the nasal route for systemic delivery of 
M. Vani et al., Saudi J Med Pharm Sci, Nov, 2020; 6(11): 673-685

medications. The nasal cavity contains two parts namely, the respiratory region and the olfactory region, which are responsible for the absorption of the drug into the brain or blood. Along the respiratory region mucosa, some compounds can enter the systemic circulatory system and subsequently cross the BBB to the brain, while some can be directly transported to the brain via the trigeminal nerve pathway or via lamina propria adsorption from perivascular and lymphatic spaces. Through the olfactory mucosa, compounds can be transported into the olfactory bulbs and then into the cerebrospinal fluid through lamina propria absorption, olfactory nerves, lymphatic and perivascular spaces, and the trigeminal nerve pathway. The olfactory mucosa is the most rapid and main pathway that mediates drug delivery from the nasal cavity to the brain. When a drug formulation is administered, it comes in contact with mucosa and gets directly transported into the brain bypassing the $\mathrm{BBB}$, thereby attaining excellent bioavailability, reduced dose and side effects $[4,5]$. Nose-to-brain drug delivery is a new advancement in drug delivery and therapeutics that holds great promise for achieving the goal of controlled and site-specific drug delivery.

\section{Blood Brain Barrier (BBB)}

Blood vessels are crucial to deliver oxygen and nutrients to all the tissues and organs throughout the body, the blood vessels that vascularise the CNS is termed as blood brain barrier (BBB). The BBB is a semipermeable membrane, comprised of capillary endothelium (CNS endothelial cells) lined with tight junctions (unlike most of the systemic circulation), and functional, with a variety of active transport mechanisms. It is also comprised of pericytes, astrocytes and neurons $[6,7]$. The endothelial cells are the first line of defence against circulating factors of the brain, these cells are different from peripheral endothelial cells in that they express tight junction proteins, creates a stronger barrier, and causes decreased pinocytosis. The endothelial cells can readily transport proteins and molecules in and out of the brain more efficiently by making up the largest surface area at the blood CNS interface [8]. Pericytes are enveloping brain micro vessels and capillaries that control cerebral blood flow by regulation of capillary diameter through actin fibres in the pericytic cell body [9]. The astrocytes in the BBB allows the blood vessels to tightly regulate the movement of ions, molecules and cells between the blood and the brain, this meticulous control of CNS homeostasis allows for proper neuronal function and also shield the neural tissue from toxins and pathogens [10]. The physiological barrier is coordinated by a series of transport (with specific transporters present on luminal and albuluminal membranes) and metabolic properties possessed by endothelial cells that forms the walls of the blood vessels. The transport and metabolic properties of the endothelial cells are regulated by interactions with different vascular, immune and neural cells [11].

\section{Drug Transport Mechanism from Nose to The Brain}

The transport mechanism of drug from nose to brain involves three different ways such as olfactory pathway, trigeminal nerve pathway, and systemic pathway (Fig-1).

\section{Olfactory Pathway}

The olfactory region of the nasal cavity remains directly connected to the frontal cortex; especially olfactory bulb of the brain via olfactory nerves. The middle and the largest region of the nasal cavity (the respiratory region) remain supplied with the trigeminal sensory neurons and blood vessels. When the drug is administered into the nasal cavity, the drug has to travel to the olfactory mucosa to undergo mucociliary clearance in the vestibular region. Olfactory mucosa contains olfactory receptor neurons responsible for the transduction in olfactory receptors on the cilia which is the end of the olfactory receptor neurons. The drug molecules reach the olfactory receptor neurons by paracellular or transcellular mechanism [12]. Moreover, the drug molecules reach to the internal portion of the nasal cavity, thereby comes in contact with the blood vessels (respiratory epithelium) and neuronal network (olfactory and respiratory epithelium). The drug enters into the systemic circulation from the blood vessels, and distributed throughout the body as per the relative volume of distribution. This systemic bioavailability remains as the minor route of drug transport to the brain via $B B B$ [7]. Drug moieties travels along axon and via nerve bundle cross the cribriform plate and reach the olfactory bulb of the brain. From the olfactory nerves, the therapeutic moiety can enter the cerebrospinal fluid (CSF) and olfactory bulb [13]. The drug can be disseminated from the CSF to brain by mixing with interstitial fluid in the brain. Following the intranasal administration, the drug takes only few minutes to reach the brain via olfactory transport. Intra-neuronal and extra-neuronal are the two different pathways of the olfactory neuronal pathway into the brain. Intraneuronal pathway involves axonal transport to reach different zones of the brain and it requires hours to days for active moiety to reach the brain. Whereas, in case of extra-neuronal pathway, the transport of active moiety takes place within few minutes through the perineural channels to reach the brain $[14,4]$. The olfactory neuronal pathway innervates to the deeper areas of brain such as cortex, cerebrum and cerebellum.

\section{Trigeminal Pathway}

The drug is transported through nose via trigeminal nerve pathway by intracellular transport or by endocytosis. The ophthalmic and maxillary branches of the trigeminal nerve $\left(5^{\text {th }}\right.$ cranial nerve) plays an 
important role in nose to brain drug delivery, the neurons from these branches pass directly through the nasal mucosa. The ophthalmic branches of the trigeminal nerve innervate the dorsal part of the nasal mucosa and the anterior nose, the maxillary branch innervates the turbinates of the nasal mucosa [13]. Once the compounds dispersed through the mucosa of the nasal cavity, it arrives at the branches of trigeminal nerves in olfactory and respiratory regions, and via brain stem, it is transported to the axonal route. A part of the trigeminal nerve that passes through the cribriform plate that may also involve in the delivery of therapeutics from nasal cavity to the forebrain $[15,16]$. Intranasally administered drug/nanoparticles absorbed from nasal cavity has to pass through the mucus. After passing through the mucus, the transportation through mucosa involves several mechanisms such as are paracellular, transcellular, carrier mediated transport, receptor-mediated transport and transcytosis. Paracellular route involves the transport of molecules between the cells. Transcellular route is attributed to the transport of drug across the cells by carrier-mediated transport or endocytosis. In transcellular route, the adsorptive transcytosis mechanism involves transport of macromolecules. This process involves interaction between the ligand in blood stream and cell surface, which may be due to electrostatic interaction between the positively charged ligand such as protein or macromolecules and negatively charged membrane. Nanoparticles and some compounds undergo transcytosis for the permeation [17].

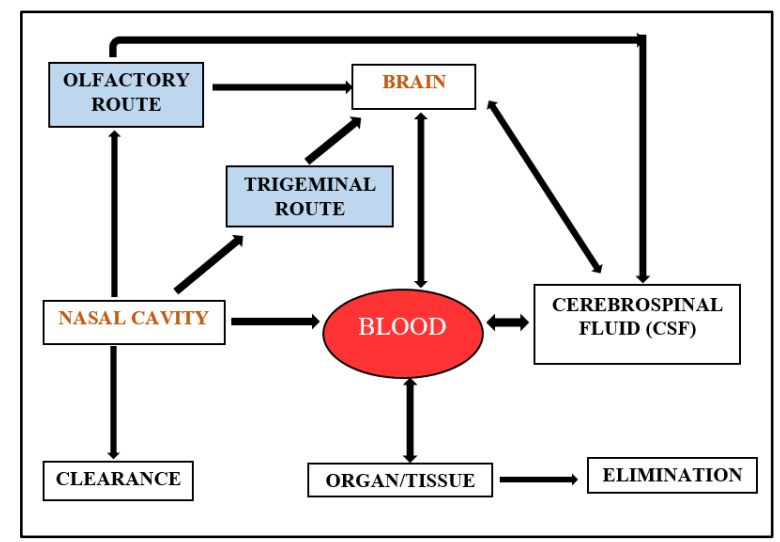

Fig-1: Drug Transport Mechanism of Nose to Brain

\section{Systemic Pathway}

The drug uptake into the brain occurs via blood circulation from nasal cavity. Due to the rich vasculature of the respiratory epithelium, a fraction of the drug is also absorbed into the systemic circulation. The respiratory segment comprising of the continuous and fenestrated endothelium allows the passage of both small and large molecules into the blood circulation and subsequently transport across the BBB to the CNS. Smaller lipophilic molecules can easily enter into the blood and cross the BBB compared to the higher molecular weight and hydrophilic molecules.
The transport mechanism of drug from nose to brain depends upon various factors including nature of the drug, type of delivery system or dosage form, a device used for intranasal application, formulation parameters, experimental and physiological conditions. It is observed that the excipients added to the formulation improves the drug retention time (like a gelling agent, and mucoadhesive polymers), a permeation enhancer, and the drug carrier system significantly affects the drug concentration in the brain. Further, the drug absorption through the neuronal pathway happens more instantly, only if the formulation remains targeted to the posterior upper region (olfactory region) of the nasal cavity $[18,19]$.

\section{Emerging Strategies to Bypass The BBB}

Drug targeting to the brain appears to be a big challenge because many of the drugs do not cross BBB. Therefore, many efforts have been made for designing the strategies to improve the penetration of the BBB and to identify the solutions to the problem of brain drug delivery. The use of the nose-to-brain delivery route is a noninvasive and an important method of drug delivery to bypass the BBB.

\section{Nanoparticle Carriers}

Oral and intravenous route of administrations face the challenge of crossing the BBB by demonstrating decreased bioavailability and lower drug concentration in the brain, thereby leading to compromised efficacy. Nanotechnology has emerged as a promising tool to tackle these problems by providing a novel platform to improve the transport of drugs across the physiological barrier. Nanoparticles (promising carriers) have become the major focus of drug delivery research for drug delivery to the brain due to unique characteristics such as small size, enhanced drug solubility, the ability for multi-functionality, a controlled drug release profile, and the potential for site-specific targeting [20]. The nanoparticle surface can be readily modified to incorporate the targeting ligands to bind receptors and transporters at the BBB enables them to bypass receptor mediated endocytosis to improve CNS selectivity and permeability. Various classes of nanoparticles including polymeric, metallic and lipid nanoparticles can also enter the brain and cross the BBB through different endocytotic mechanisms [21].

\section{Liposomes}

Liposomes are spherical, colloidal structure and self-assembled vesicles made of amphiphilic phospholipids that mimic the lipid bilayer of the cell membrane. Liposomes (biocompatible carriers) are capable of carrying hydrophilic, hydrophobic, and amphoteric drug molecules. Liposomal formulations are a well-known novel drug delivery system which have been used to deliver growth factors and specific 
proteins to the brain due to their resemblance to the lipid bilayer of the endothelial cell membrane. The basic mechanisms for liposomal transport across the BBB include adsorptive-mediated transcytosis (AMT) and receptor-mediated transcytosis (RMT) [22]. Although the lipophilic nature of liposomes has enabled the transport of hydrophilic and hydrophobic drug molecules across the various biological membranes, the BBB remains the major obstacle that prevents their penetration into the brain. Modification of liposomes surface facilitates the permeation of liposomes into the brain through receptor-mediated endocytosis by enabling the targeting of receptors on the BBB [21, 23]. Liposomes are considered to be more potent in treating the neurodegenerative disorders, but may face challenges such as rapid elimination from the blood and accumulation in spleen and liver, get degraded by the GIT enzymes and poor permeability of liposomes across GI epithelia, difficulties in sterilization and poor storage stability [24].

\section{Metallic Nanoparticles}

Metallic nanoparticles are commonly made of inorganic materials and include gold, silver, and iron oxide particles, since they are typically smaller than polymeric or lipid nanoparticles, they have an advantage with regard to crossing the BBB. Metallic nanoparticles can be transported into the brain through several routes, including passive diffusion, carriermediated transport (CMT), or transsynaptic transport. Drugs cannot be encapsulated within the metallic nanoparticles due to their solid and dense structure. The anticancer drugs, antibodies, and siRNA are some of the therapeutic agents that can undergo conjugation to the surfaces of these nanoparticles. A few in vivo studies have shown that metallic nanoparticles, including those nanoparticles comprised of silver, gold and iron oxide can cross the BBB [25]. Kong et al., [26] in his study demonstrated that following the systemic administration of magnetic iron oxide nanoparticles and application of an external magnetic field, the nanoparticles can cross the BBB and accumulate in a perivascular zone via a transcellular mechanism. Cheng et al., [2] conducted a study and demonstrated that gold nanoparticles of size $5 \mathrm{~nm}$ in diameter when modified with the transactivator of transcription peptide are capable of crossing the BBB and delivering both gadolinium and doxorubicin contrast agents to a murine intracranial glioma xenograft.

\section{Polymeric Nanoparticles}

Polymeric nanoparticles are colloidal systems which have an ability to load drugs in solid-state or solutions, chemical grafting on the surface or noncovalent adsorption. Polymeric nanoparticles being less rigid and less dense, enables them to encapsulate a wide variety of therapeutic agents including chemotherapeutic drugs, proteins, nucleic acids, and contrast agents. Polymer-based delivery systems offer a number of advantages such as low toxicity of degradation products, biocompatibility, enhanced absorption, sustained release compatibility, ease of production, and localized drug delivery. Biocompatibility and rapid biodegradability of the applied polymer are considered major advantages in transporting nanoparticles in brain delivery systems [28]. Nanoparticle surface modification with adsorption or chemical-linking of polyethylene glycol (PEG), or with targeting ligands can provide an optimal way to prolong their circulation time and facilitate drug delivery across the BBB. Several studies report that PEGylated nanoparticles cross the BBB more efficiently than other nanoparticles with various other surface modifications, although the mechanism of BBB penetration by PEGylated nanoparticles is not clear. The major obstacle encountered in the use of polymeric nanoparticles is the interaction between the carrier and the reticuloendothelial system (RES), thereby exhibiting a rapid clearance from blood circulation. Therefore, lipid nanoparticles propose an alternative strategy for drug delivery to the brain [29].

\section{Micelles}

Polymeric micelles are composed of a block copolymer with amphiphilic properties. This colloidal carrier system acts as a novel drug delivery system to provide the possibility of high drug-loading efficacy, like other colloidal delivery systems polymeric micelles suffer from the issue of uptake of reticuloendothelial systems affecting their circulation period in the blood. The determinant factors to design the desirable drug delivery systems are the size and surface properties of polymeric micelles. Surface functionalization of these polymeric micelles has an advantage of receptor mediated drug delivery to a specific site of the brain.

In a study conducted by Xiaonan Tan et al., [30] investigation on the combination therapy of curcumin and an antisense-oligonucleotide against miR21 (miR21 ASO) for the treatment of glioblastoma was carried out using deoxycholic acid-conjugated polyethyleneimine (DP) micelles. The curcumin-loaded DP micelle (DP-Cur) was evaluated as a carrier for the combined delivery of curcumin and miR21ASO. The enhanced drug penetration of this formulation to the C6 glioblastoma cells could be attributed to the interaction of the positive surface charge of DP-Curcumin micelles with the negatively charged cell membranes, which consequently trigger the cell internalization of curcumin via an endocytosis pathway. In the C6 cells, the delivery of miR21ASO using DP-Cur effectively reduced the miR21 level in the brain tumor.

\section{Lipid Nanoparticles}

Lipid nanoparticles(NPs)are the colloidal systems which are preferred over other traditional colloidal carriers (liposomes, emulsions, and polymeric micronanoparticles) because of their properties like 
lipophilic nature, controlled release potential, high encapsulation properties, drug targeting and the possibility of incorporating hydrophilic and lipophilic drugs - all of which make lipid NPs the viable candidates for brain delivery of therapeutic agents. AD poses an increasing challenge to scientists and health care providers worldwide, as many decades of research in the medical field revealed no optimal effective treatment for this disease. The intranasal administration route seems to be the preferable route for anti-AD drug delivery over the oral one because of its ability to overcome the obstacles which are reflected in limited brain exposure, low bioavailability, and undesired pharmacokinetics or side effects. The intranasal drug delivery route through the intraneuronal and extra neuronal pathways can bypass the systemic circulation, thereby provides truly needleless and direct brain drug delivery of the therapeutics due to its large surface area, porous endothelial membrane, the avoidance of the first-pass metabolism, and ready accessibility. In contrast to the nano-carrier systems developed, lipidbased nanosystems have proved to be more effective and have become increasingly popular in managing the common symptoms of $\mathrm{AD}$ when administered via the nose-to-brain delivery route [31].

\section{Recent Examples of Nose to Drug Targeted Therapy} Numerous medications for intranasal administration are still in the preclinical phase of development while some of the agents are being investigated in clinical trials. Mainly targeting to demonstrate the potential treatment for neurodegenerative disorders like $\mathrm{AD}, \mathrm{PD}$, epilepsy, neurotoxic events like oxidative stress and ischemia, and other diseases as it can be seen in Table $1 \& 2$.

Coronavirus 2019 (Covid-19) is an infectious disease-causing respiratory illness. Recent studies suggest that severely ill covid-19 patients may develop neurological manifestations, in addition to pulmonary damage. In covid-19 cases with the CNS involvement, as confirmed by brainstem concentration of infection, treatment involves the intravenous (IV) injection of drugs, but even with aggressive treatment, the prognosis is often poor and there exists complexity in conveying anti-covid-19 drugs to the infected brain. Hence enhanced therapeutic effect may be accomplished via the administration of drug therapy through the nasal cavity traversing the cribriform plate to access the brain, for covid-19 cases with the CNS involvement $[32,33]$. In a prospective, open-label study by Meng Z et al., has shown that IN administration of recombinant human interferon alpha (rhIFN- $\alpha$ ) has effectively prevented covid-19 in medical staff, as an enhancement protection on the basis of standard physical isolation thus protecting susceptible healthy people during the covid-19 pandemic [34].

Table-1 Pre-clinical studies of various agents for nose to brain targeted delivery

\begin{tabular}{|c|c|c|c|c|c|}
\hline Drugs & Category & Animals & Indications & Results & References \\
\hline NAP peptide & Neuroprotective & Mouse & $\mathrm{AD}$ & $\begin{array}{l}\text { Reduced neurodegeneration; tau } \\
\text { hyperphosphorylation; Improved } \\
\text { cognitive behaviour }\end{array}$ & $\begin{array}{l}\text { Hanson LR et al., } \\
(2020)\end{array}$ \\
\hline L-DOPA & $\begin{array}{l}\text { Dopamine } \\
\text { agonist }\end{array}$ & Rat & Parkinson's Disease & $\begin{array}{l}\text { Fewer ipsilateral, contralateral } \\
\text { rotations and forelimb-slips }\end{array}$ & $\begin{array}{l}\text { Diddams M et al., } \\
\text { (2018) }\end{array}$ \\
\hline $\begin{array}{l}\text { PLGA- } \\
\text { oxcarbazine }\end{array}$ & Anticonvulsant & Rat & Seizure & Reduced seizure activity & $\begin{array}{l}\text { Musumeci et al., } \\
\text { (2018) }\end{array}$ \\
\hline EPO & HGF/ Hormone & Mice & Neurologic dis & $\begin{array}{l}\text { Reduce neurodegeneration \& delay } \\
\text { deterioration onset in amyotrophic } \\
\text { lateral sclerosis }\end{array}$ & Nie X et al., (2018) \\
\hline $\begin{array}{l}\text { Orexin-A/ } \\
\text { Hypocretin }\end{array}$ & Neuropeptides & Rat & $\begin{array}{l}\text { Cognitive disorder; } \\
\text { Obesity }\end{array}$ & $\begin{array}{l}\text { Enhanced age-related cognitive } \\
\text { disorder } \\
\text { Suppression of food intake }\end{array}$ & $\begin{array}{l}\text { Calva CB et al., } \\
\text { (2020); Steward T } \\
\text { et al., (2019) }\end{array}$ \\
\hline Leptin & Hormone & Mice & $\begin{array}{l}\text { OSA; Morphine- } \\
\text { induced } \\
\text { hypoventilation }\end{array}$ & $\begin{array}{l}\text { Reversed the opioid induced } \\
\text { depression of excitatory synaptic } \\
\text { neurotransmission }\end{array}$ & $\begin{array}{l}\text { Freire C et al., } \\
(2020)\end{array}$ \\
\hline MSC & Stem Cell & Mouse & Neurotrophic factor & Induced recovery by cerebral repair & $\begin{array}{l}\text { Nijboer et al., } \\
\text { (2018) }\end{array}$ \\
\hline
\end{tabular}

Abbreviations: NAP-Neuroprotective Protein, PLGA- Poly(L-lactide-co-glycolide), L-DOPA- Levodopa, EPO- Erythropoietin, OSAObstructive Sleep Apnoea, MSC- Mesenchymal Stem Cells 
Table-2: Clinical studies of various agents for nose-to-brain targeted delivery

\begin{tabular}{|l|l|l|l|l|}
\hline Drugs & Category & Indications & Result & References \\
\hline Diazepam & Benzodiazepines & Epilepsy & Acceptable safety/tolerability profile & Miller I et al., (2020) \\
\hline Insulin & Hormone & $\begin{array}{l}\text { Alzheimer's } \\
\text { disease }\end{array}$ & Improved memory \& mood & Hanson LR et al., (2020) \\
\hline Bromocriptine & Dopamine D2 agonist & $\begin{array}{l}\text { Parkinson's } \\
\text { Disease }\end{array}$ & $\begin{array}{l}\text { Reduced lipid oxidation; restored } \\
\text { GSH levels in brain }\end{array}$ & Sita VG et al., (2020) \\
Covid-19 & $\begin{array}{l}\text { Tyrosine phosphorylation of IFN } \\
\text { receptor in CNS } \\
\text { Improved survival }\end{array}$ & $\begin{array}{l}\text { Dixon BJ et al., (2016) } \\
\text { NCT04320238 }\end{array}$ \\
\hline Zolmitriptan & SRA (1B \& 1D) & Migraine & $\begin{array}{l}\text { Higher retention effects in desired } \\
\text { areas }\end{array}$ & NCT03708744 \\
\hline Oxytocin & Peptide Hormone & $\begin{array}{l}\text { Social cognitive } \\
\text { disorders }\end{array}$ & Enhanced social cognitive functions & Quintana DS et al., (2020) \\
\hline Desvenlafaxine & SNRI & Depression & $\begin{array}{l}\text { Enhanced PK profile of } \\
\text { desvenlafaxine in brain }\end{array}$ & Tong GF et al., (2017) \\
\hline $\begin{array}{l}\text { Orexin - } \\
\text { A/Hypocretin }\end{array}$ & Neuropeptide & Narcolepsy & $\begin{array}{l}\text { Stabilizes REM sleep and reduces } \\
\text { wake-up frequency }\end{array}$ & Barateau L et al., (2019) \\
\hline
\end{tabular}

Abbreviations: IFN-Interferon beta; SRA- Serotonin Receptor Agonist; GSH- Glutathione; NCT-National Clinical Trial; SNRI- Serotonin and norepinephrine reuptake inhibitors; PK- Pharmacokinetics REM- Rapid Eye Movement

\section{Applications of Nanocarrier Technology for Nose to Brain Drug Delivery Targeting Brain Gliomas \\ Gliomas or tumors arising from glial cells are} devastating diseases accounting for over $75 \%$ of malignant adult brain tumors despite of development of drugs that preferentially targets tumor cells without harming normal tissues. According to world health organization (WHO) classification for CNS tumors, Glioblastoma multiforme (GBM) is classified as highest (i.e. grade IV) and is the most common aggressive form of malignant primary brain tumor in adults whereas in children, it is considered to be the second leading cause of death even with surgery followed by radiation therapy and chemotherapy [35]. Current treatments for GBM remain palliative rather than curative; therefore, innovative delivery strategies are required, of which nanoparticles (NPs) are at leading edge of future solutions [36].

There are many challenges associated with systematically delivered chemotherapeutics including the limited brain penetration, low bioavailability, rapid degradation and high efflux from the brain. To improve bioavailability and enhancing the nose-to-brain drug delivery, the most common method in biomedical application is to encapsulate chemotherapeutics in appropriate carrier systems such as nanoparticles, microspheres, or lipid-based nanosized formulations for controlled release and targeted delivery [37].

Nanoparticles have the potential to improve nose-to-brain delivery as they avoid enzymatic degradation and transport from P-glycoprotein (P-gp) efflux proteins, thus promising a prime choice for targeting life-threatening diseases like GBM. Novel delivery approaches using liposomes, nanostructured lipid carriers (NLCs), solid lipid nanoparticles (SLNs), lipoproteins, lipoplexes and nanostructured lipid carriers have been widely used for targeting GBM.
Among all other lipid nanoparticles, SLNs and NLCs are the most suitable without being constrained by their limitations. The biodistribution study of Curcuminloaded NLCs (CUR-NLC) showed an increased drug concentration in the brain after intranasal administration. Similarly, the in vivo studies indicated enhanced brain concentration of novel Temozolomideloaded NLCs (TMZ-NLC) and farnesyl thio salicylic acid (FTA)-loaded lipid cationic hybrid nanoparticles (HNPs) in comparison with its dispersion (IV). The study also described that the intranasal administration of NLCs increased residence time and resulted in higher bioavailability in the brain at lower doses, denoting most suitable delivery route for targeting glioma. Furthermore, these lipid nanoparticles have a good ability to protect the loaded active pharmaceutical ingredient (API) and because of their occlusive nature, they can also increase nasal retention time. These features make lipid nanoparticles a significant carrier for nose-to-brain delivery as they avoid the cytotoxicity issues of antineoplastic drugs [38].

An excellent study by Hashizume R et al., in 2008, showed a favourable result of GRN163, a 13-mer oligonucleotide $\quad \mathrm{N} 3^{\prime} \rightarrow \mathrm{P}^{\prime} \quad$ thio-phosphoramidate telomerase inhibitor by targeting tumor-specific malignant gliomas. The enzyme telomerase, which is expressed in majority of GBMs while not in normal brain tissues, thus the inhibition of telomerase provides a therapeutic strategy for the treatment of intra-cerebral tumors. Moreover, intranasal administration of GRN163 in contrast to convection-enhanced delivery (CED) which involves invasive procedures, has more advantage of being non-invasive and without neurotoxic side effects [39].

In a study conducted by Jain and collaborators, developed an innovative methotrexate (MTX) formulation for GBM by encapsulating the drug into 
polymeric PLA nanoparticles (MTX-NP) and including poloxamer 188 in coalition with Carbopol 934 in the formulation to obtain a thermosensitive hydrogel. In vivo studies carried out using male Wistar rats indicated that combination of the in-situ gelling system and nanoparticles resulted in an increase of MTX in the brain when compared to data obtained with MTX solution.

Gadhave and his team worked on microemulsion (ME) and mucoadhesive hydrogel (MME) for intranasal delivery of teriflunomide (TFM) with the aim to increase its delivery to brain. The teriflunomide-mucoadhesive hydrogel (TFM-MME) formulation showed enhanced brain accumulation with a direct transport percentage (DTP) of $99.2 \%$ and a brain targeting efficiency (DTE) of $359 \%$ when compared with the intravenous TFM-ME. Although these formulations demonstrated to be safe for nasal administration, the study needs more robust in vitro and in vivo investigations to demonstrate the efficacy of the and TFM-MME to treatment of GBM. In a similar study, another group developed a polymeric nanoparticle (NP) formulation of carboplatin (CPC) using the biodegradable polymer poly ( $\varepsilon$-caprolactone). Carboplatin (CP) is an antineoplastic drug belonging to the class of platinum-based alkylating agents and is widely used to treat various forms of cancer. However, there are some challenges related to CPC use in oncology practice such as development of resistance, systemic toxicity and rapid blood clearance. In vitro cytotoxicity study on GBM cells revealed that CP nanoencapsulated drug showed better nasal absorption compared to free drug after IN administration [40].

Faria et al., demonstrated a study indicating that intranasal perillyl alcohol $(\mathrm{POH})$ therapy mitigate the detrimental effects of global gDNA hypomethylation on tumor cell proliferation and improved survival of patients with recurrent glioblastoma (rGBM) harboring TT mutant variant for methylenetetrahydrofolate reductase (MTHFR) rs1801133 polymorphism [41]. Although, $\mathrm{POH}$ has been undergoing clinical trials under investigational drug (IND) approval for rGBM.

Recently, Van Woensel and collaborators formulated chitosan nanoparticles with a galectin 1 (Gal-1)-siRNA for intranasal delivery to treat GBM in mice models. Gal-1 is a protein over-expressed in GBM and highly associated with tumor progression. The intranasal delivery of Gal-1 siRNA induced an exceptional switch in the tumor micro-environment cellular composition, reducing macrophage polarization from M1 (pro-inflammatory) to M2 (anti-inflammatory) and thus inhibiting recruitment of monocytic myeloid derived suppressor cells amid GBM progression.

Stem cells approach for GBM treatment has also been coupled with nanotechnology. Stem cells can be derived from multipotent stem cells including mesenchymal stem cells (MSCs) and neuronal stem cells (NSCs). MSCs are hematopoietic stem cells and can be isolated from different tissue sources, such as adipose tissue or bone marrow, making them easier to isolate than NSCs. MSCs have the capability to selfrenewal, to differentiate in specific functional cellular and immune-compatible nature. Mangraviti and coworkers developed a system combining polymeric nanoparticles and human adipose tissue derived MSCs to deliver bone morphogenetic protein 4 (BMP4) and evaluated the antitumor effect in a primary malignant glioma model. Thus, MSCs transfected with polymeric nanoparticles to express BMP4 administered via IN in rats significantly improved the survival of tumor bearing animals [41].

In spite of 40 years of work in trans-nasal drug delivery to the brain, various drugs are still under investigation to be approved by FDA for the treatment of human brain through intranasal delivery as listed below (Table-3) [42].

Table-3: List of investigational intranasal drugs using nanocarriers for GBM therapy

\begin{tabular}{|l|l|l|l|l|}
\hline $\begin{array}{l}\text { Encapsulated } \\
\text { Agents }\end{array}$ & Type of Nanocarriers & Evaluation & Result & Reference \\
\hline Temozolomide & NLC & Clinical & Enhanced antitumor activity & Lynn J et al., (2020) \\
\hline Curcumin & $\begin{array}{l}\text { RDP-Conjugated } \\
\text { liposomes (RCL) }\end{array}$ & Clinical & $\begin{array}{l}\text { Inhibiting U251MG cells growth by } \\
\text { apoptosis induction and cell cycle } \\
\text { arrest at the S phase }\end{array}$ & Zhao et al., (2018) \\
\hline Doxorubicin & PEG-liposomes & Clinical & $\begin{array}{l}\text { Enhanced efficacy Inhibition of tumor } \\
\text { growth }\end{array}$ & Beier CP et al., (2009) \\
\hline $\begin{array}{l}\text { Daunorubicin \& } \\
\text { quinacrine }\end{array}$ & Ligand-targeted liposomes & Pre-clinical & $\begin{array}{l}\text { Killing of glioblastoma cells and } \\
\text { diminishing brain gliomas }\end{array}$ & Li et al., (2014) \\
\hline $\begin{array}{l}\text { Interferon } \\
\text { (IFN)-beta }\end{array}$ & Cationic Liposomes & Clinical & $\begin{array}{l}\text { Antiproliferative Reduction of tumor } \\
\text { size }\end{array}$ & Li G et al., (2017) \\
\hline $\begin{array}{l}\text { Irinotecan, } \\
\text { Curcumin }\end{array}$ & PEGylated Nano diamonds & Pre-clinical & $\begin{array}{l}\text { Cytotoxic effect on tumor cell with } \\
\text { minimized systemic toxic effects }\end{array}$ & Madamsetty VS et al., (2019) \\
\hline Cytarabine & NLCs & Pre-clinical & Cytotoxic effect on tumor cell line & Sharma P et al., (2011) \\
\hline SiRNAs & LDL \& PEG SLNs & Pre-clinical & The decrease in tumor cell proliferation & Jin et al., (2011) \\
\hline Bevacizumab & Nanoemulsion PLGA NPs & Pre-clinical & Decreased angiogenic profile & Tang W et al., (2019) \\
\hline MTX & PLA & Preclinical & Increase of MTX & $\begin{array}{l}\text { Sedeky AS } \\
\text { et al., (2018) }\end{array}$ \\
\hline
\end{tabular}


Abbreviations: NCL-Nanostructured lipid carriers; PEG-Polyethylene glycol; LDL-Low density lipoprotein; SLN-Solid lipid nanoparticles; PLGA-Poly(lactic-co-glycolic acid); NP-Nanoparticles; PLA-Poly(lactic acid); MTX-Methotrexate

\section{Applications of Nose to Brain Targeted Therapy for Neurological Disorders}

In modern medicine, the intranasal drug delivery is emerging as a non-invasive option for various neurological dysfunctions with minimal peripheral side effects. Therefore, this method facilitates the delivery of compounds such as growth factors, hormones, neuropeptides, and therapeutics including insulin, oxytocin, orexin, stem cells for the treatment of various neurological disorders.

\section{Parkinson's Disease}

Parkinson's disease (PD) is the second most noted cause of dementia and is characterized by a dynamic loss of dopaminergic neurons in the substantia nigra along with the presence of intraneuronal $\alpha$ synuclein-positive inclusions. PD leads to an array of symptoms including progressive loss of motor functions with bradykinesia, gate alterations, and posture instability [43]. The progression of PD can be deferred by levodopa - the precursor of dopamine-or a levodopa agonist. However, it has been demonstrated that untargeted delivery of levodopa can penetrate the peripheral nervous system, thus leading to dyskinesia and adverse cardiovascular effects. Several studies are exploring various drugs such as Ropinirole, Bromocriptine, Mitoapocynin, Apomorphine, when encapsulated with liposomes or polymeric nanoparticles have shown to enhance sustained release of drugs and to reduce undesired effects of empiric PD therapy [44]. Therefore, it is advisable to carefully deliver neurotransmitters for PD treatment across the BBB by using a suitable delivery system, which does not allow penetration into other peripheral vessels [45]. In a study Tang et al., demonstrated a novel drug delivery system by developing a borneol and lactoferrin co-modified NPs (Lf-BNPs) encapsulating dopamine to achieve maximal therapeutic efficacy and reduced side effects for PD in a rat model. Pharmacokinetic studies showed a significant increase in the absorption of dopamine LfBNPs into the brain after nose-to-brain delivery when compared to that of dopamine NPs, which demonstrated that borneol and Lf co-modification could together enhance drug transport to the brain via intranasal administration [46].

Insulin plays a vital role in glucose metabolism in the brain where it exerts neurotrophic, neuroprotective, and neuromodulatory effects. A pilot longitudinal study conducted by Novak P et al., has shown that intranasal insulin (INI) administration may improve functional motor skills in patients diagnosed with PD and may potentially preserve cognitive performance in contrast to baseline and the placebo group. However, a longer duration of treatment may be needed to achieve long-term effects [47].

\section{Alzheimer's Disease}

Alzheimer's disease (AD) is the main form of dementia in the elderly, thus urgently seeking for a cure. Patients diagnosed with AD show deposited extracellular senile plaques enriched in $\beta$-amyloid (A $\beta$ ) aggregates, and intracellular tau-enriched neurofibrillary tangles in brain [48]. In a 4-month study by Craft et al., 2017, compared intranasal long-acting insulin - insulin detemir (insulin with a C14 fatty acid chain at the proline residue) with regular insulin, in which patients received a daily dose of 40 IU insulin, and found memory improvements at months 2 and 4 in the regular insulin group and not in the insulin detemir group. The potency of insulin to translocate and interact with the relevant brain regions was examined further by using rapidly acting insulins such as insulin aspart (does not form hexamers) and regular insulin (forms hexamers). The datas show the nonaggregated insulin available as monomers and not as hexamers or the lipidized analog is more efficient at locating relevant brain receptors when dosed via the nose-to-brain route [49].

In the present scenario, extensive research are undergoing using the IN route on various agents including galantamine, donepezil, rivastigmine, risperidone, tacrine, deferoxamine, tarenflurbil, insulin, insulin-like growth factor, curcumin, piperine, quercetin, NGF, vasoactive intestinal peptide (VIP), bFGF, erythropoietin, wheat germ agglutinin, H102 peptide, V24P, melanocortins, various anti-amyloid $\beta$ antibodies, stem cells for nerve regeneration and various genes or genetic factors for the management of the AD. Among these, only two drug substances (insulin and rivastigmine) are under clinical investigation while rest are still under preclinical examination. Though, the preclinical studies of various drugs (donepezil, curcumin, galantamine, risperidone, tacrine, memantine, and quercetin) and peptides (NGF, bFGF, VIP, S14G-humanin, and melanocortins) on different animal models with $\mathrm{AD}$ demonstrated improved memory, cognition and behavioural functions of the animal [50].

\section{Epilepsy}

Epilepsy is the fourth most common global neurological problem after migraine, stroke, and $\mathrm{AD}$ as reported by the WHO in its report "Epilepsy" in April 2018. It is characterised by recurrent seizures or/and periods of strange behaviour, sensations, and sometimes loss of consciousness. Nasal therapy could be a most sensible solution to treat seizure emergencies. Currently, there are benzodiazepines including lorazepam (LZP), diazepam (DZP), and midazolam (MDZ) nasal spray clinical trials underway for people with seizures. As reported in the literature, intranasal 
M. Vani et al., Saudi J Med Pharm Sci, Nov, 2020; 6(11): 673-685

MDZ is safe and effective for treatment of acute seizures in children, and quicker than intravenous DZP to stop seizures. Another study performed by Westin and colleagues depicted that morphine can be transferred along the olfactory pathway to the CNS surrounding the olfactory bulbs in rats within five minutes of IN administration. Similarly, another interesting result shown by Veronesi et al., intra-nasally administered thyrotropin-releasing hormone (TRH) demonstrated reduced seizure in animal models. In a comparison study between intranasal (IN) and intraperitoneal (IP) pentoxifylline by Kang et al., it was found that IN delivery of pentoxifylline significantly suppressed the epileptic seizures induced by lithiumpilocarpine, ameliorated the deficits in visuospatial memory and in the mesodopaminergic system, and enhanced the transient activation of nuclear factor erythroid 2-related factor 2-(Nrf2-) in status epilepticus rats. Over the next few years, there is an expectation that the US Food and Drug Administration (FDA) will approve a BZP nasal spray and make it available to people with epilepsy [51].

\section{Multiple Sclerosis}

Multiple sclerosis (MS) is a chronic demyelinating disorder of the CNS. Formation of demyelinating lesions in the brain and spinal cord causing axonal damage is the pathological hallmark of MS, leading to neuropsychiatric problems [44]. Teriflunomide (TFM) is an effective inhibitor of dihydroorotate dehydrogenase (DHODH) has been approved by the FDA for the treatment of MS. In a vivo sub-acute toxicity study by Gadhave DG et al., confirmed that IN administration of TFM-loaded NLC (TFM-NLC) rapidly achieved at the target site and reduced the neuroinflammatory pathways as compared to oral administration of TFM-NLC. Hence, TFM-NLC was found to be safe and effective in the treatment of MS as well as it reduces the risk of hepatotoxicity caused by oral administration [52]. Similarly, in a study by Rassy D et al., revealed that IN administration of methylprednisolone in mice models, suppressed the neuroinflammatory peak and reduced immune cell infiltration \& demyelination in the CNS, indicating that IN delivery is as efficient as IV route in case of relapsed MS [53]. A study by Khan RS et al., demonstrated that IN administration of a novel amnion cell secretome suppressed inflammation, prevented neuronal damage and preserved neurologic function in the experimental autoimmune encephalomyelitis animal model of multiple sclerosis [54]. Preclinical trials have already produced promising results; however, many more trials are still needed to demonstrate the safety and efficacy of these approaches.

\section{Ischaemic Stroke}

Ischemic stroke is still one of the leading causes of death across the globe. Studies have described that IN delivery of multiple substances can effectively prevent the ischemic brain injury in animal models including stem cells, gene vectors and nanoparticles, proteins and peptides,. Administration of insulin-like factors (IGF-1) by the IN approach has significantly alleviated infarct volume and improved motor-sensory and somatosensory functions in rats. Additionally, after middle cerebral artery occlusion (MCAO), the intranasal IGF-1 decreases neuronal apoptosis within the ischemic ipsilateral hemisphere. Similarly, IN delivery of granulocyte colonystimulating factor (G-CSF) also decreased infarct volume, increased recovery of neurological function, promoted angiogenesis and neurogenesis following ischemia in rats. Intranasal administration of exendin4 mediated by anti-apoptotic mechanism in MCAO mice, exerted a neuroprotective effect and protected neurons against ischemic injury through the glucagonlike peptide 1 receptor (GLP-1R) pathway. Recent studies have shown that IN administration of bone marrow mesenchymal stem cells (BMSCs) and MSC over-expressing brain-derived neurotrophic factor (MSC-BDNF) has neuroprotection and great potential as a regenerative therapy to enhance neurovascular regeneration after neonatal stroke in rats. Another study has revealed that IN administration of Salvinorin A (a kappa opioid receptor (KOR) agonist) could reduce the infarct volume and improve the neurological outcome via inhibiting the apoptosis and the inflammation. Furthermore, novel drug formulations for effective brain targeting and clinical usage have to be developed [55].

\section{Psychotic Disorders}

Autonomic nervous system (ANS) dysfunction (i.e., increased sympathetic and decreased parasympathetic activity) has been proposed to contributing factor to psychosis vulnerability. Psychotic disorders are one among the world's leading causes of disability. Currently, there is no effective intervention that can make an impact on transition to psychosis. Therefore, novel treatments for such population are urgently needed. Oxytocin has been considered to be the most promising compound in treating (both positive and negative) symptoms of psychosis. Research has shown that elevated oxytocin concentrations in cerebrospinal fluid (CSF) after IN administration in rodents, macaques, and humans. Increase in CSF oxytocin concentrations after intranasal oxytocin administration appear to be accompanied by the cocomitant increase in peripheral levels. A study examining the dose-dependent effects of IN and IV oxytocin administration on CSF oxytocin levels in macaques found that IN dose $(5 \mathrm{IU} / \mathrm{kg})$ increased $\mathrm{CSF}$ oxytocin levels, despite no increase in plasma oxytocin levels [56]. In a double-blind, randomized, placebocontrolled, parallel design study by Parker KJ et al., the efficacy and tolerability of 4-week IN administration of arginine vasopressin (AVP) to children with ASD aged 6 to 12 years was assessed. It was found that AVP 
compared to placebo treatment improved social abilities in children with Autism spectrum disorder (ASD). These preliminary findings suggest that IN-AVP may be a promising medication for treatment of core social impairments in children with ASD [57]. A similar study has shown that IN ketamine has proven to reduce the core social deficits of ASD, however, it has yet to be explored through clinical trials in ASD [58]. Risperidone loaded chitosan nanoparticles has shown to be effective against schizophrenia via IN route as per a study by Rukmangathen $\mathrm{R}$ et al [59]. Although the direct nose-to-brain delivery of antipsychotics are effective in the management of psychosis-related disorders, still there exist physicochemical challenges related to psychotropic drugs, alongside biochemical barriers associated with targeting the olfactory region.

\section{CONCLUSION}

Since there is a long list of drugs that have been developed for the treatment of CNS related disorders but due to its inability for drug substances to cross the BBB, many of them were discarded. Most of the drugs that are presently being used for brain diseases use the IV route of administration due to its rapid onset of action, however, its side effects are more pronounced as compared to other routes. Over the last several decades, majority of research and development projects are actively pursuing the development of novel nasal drug-delivery systems that are capable to pass through BBB. A number of studies on both animals and human subjects have also demonstrated that intranasal delivery of various types of drug molecules can produce salubrious effects on the brain. Although the exact mechanism of nose to brain drug delivery remains subtle, the olfactory and trigeminal nerve pathways have been found to play a vital role in circumventing the traditional barriers of brain targeting. The present outlook for patients suffering from CNS related diseases may appear feeble, but recent developments in drug delivery techniques has provided a reasonable hope in overcoming those formidable barriers.

The nose-to-brain targeted therapy present a potential approach in contrast to other routes of drug administration and is supposed to be the next generation therapy for brain \& CNS disorders despite of several limitations. After reviewing the research publications, it is evident that the application of nanocarriers to the formulation design, altering the physico-chemical properties of the drug results in higher bioavailability of drugs in pre-clinical studies. Besides many advantages, nanoformulations loaded with cytotoxic material can still accumulate in tissues of the body, for instance in the liver, spleen and kidney. Hence, nano formulations are considered as one of the most important targeting carriers that can overcome the shortcomings of previously used carriers and delivery routes. In this review, we have enlisted the different therapeutic agents that are entrapped into nanostructured carriers (solid lipid nanoparticles, polymeric nanoparticles, nanoemulsions, liposomes and polymeric micelles) have been designed and investigated for several neurological disorders to enhance nose-to-brain delivery. Moreover, the alternative route and the lipid nanocarrier provide chances to deliver anticancer drugs (with potential efficacy) in their bioactive form against GBM. Considering the facts that most of the studies are still in the preclinical stage, translating such intranasal delivery strategies would be a critical step for prognosis and better management of neurological disorders including GBM.

Recently, it has been found that intranasal drug delivery has potential relevance for future clinical trials in the settings of preventing disease transmission and treatment of SARS-CoV-2 and other viral diseases. The strategy is still under investigations with various research studies and surplus challenges to get better insight for the development of successful nose to brain targeted therapy before reaching the pharmacy counter. This article summarizes the current knowledge regarding intranasal drug delivery and its potential applications in combating several CNS disorders and viral pandemics like SARS-CoV-2.

\section{ACKNOWLEDGEMENT}

The authors wish to thank Dr. M. Vani, Professor, K.K. College of Pharmacy, for her exceptional guidance and Dr. A. Meena, Principal, K.K. College of Pharmacy, for her moral support and encouragement.

\section{REFERENCES}

1. Neurological Disorders Collaborator Group. (2015). Global, regional, and national burden of neurological disorders during 1990-2015: a systematic analysis for the Global Burden of Disease Study 2015. Lancet Neurol, 16(11), 87797.

2. Ding, S., Khan, A. I., Cai, X., Song, Y., Lyu, Z., Du, D., ... \& Lin, Y. (2020). Overcoming bloodbrain barrier transport: Advances in nanoparticlebased drug delivery strategies. Materials Today. 37,112-25.

3. Gao, H. (2016). Progress and perspectives on targeting nanoparticles for brain drug delivery. Acta Pharm Sin B, 6(4), 268-86.

4. Selvaraj, K., Gowthamarajan, K., \& Karri, V. V. S. R. (2018). Nose to brain transport pathways an overview: Potential of nanostructured lipid carriers in nose to brain targeting. Artificial Cells, Nanomedicine, and Biotechnology, 46(8), 20882095.

5. Kamble, M. S., Bhalerao, K. K., Bhosale, A. V., \& Chaudhari, P. D. (2013). A Review on Nose-ToBrain Drug Delivery. Int J Pharm Chem Sci, 2(1), 516-25. 
6. Gupta, S., Dhanda, S., \& Sandhir, R. (2018). Anatomy and Physiology of blood-brain barrier. Brain Targeted Drug Delivery System A Focus on Nanotechnology and Nanoparticulate. Elsevier, 731.

7. Crowe, T. P., Greenlee, M. H. W., Kanthasamy, A. G., \& Hsu, W. H. (2017). Mechanism of intranasal drug delivery directly to the brain. Life Sci, 195, 44-52.

8. Kashyap, K., \& Shukla, R. (2019). Drug Delivery and Targeting to the Brain through Nasal Route: Mechanisms, Applications and Challenges. Curr Drug Deliv, 16(10), 887-901.

9. Liu, Q., Yang, Y., \& Fan, X. (2020). Microvascular pericytes in brain-associated vascular disease. Biomed Pharmacother, 121, 109633.

10. Michinaga, S., \& Koyama, Y. (2019). Dual roles of astrocyte-derived factors in regulation of bloodbrain barrier function after brain damage. Int J Mol Sci, 20(3), 571.

11. Daneman, R., \& Prat, A. (2015). The blood brain barrier. Cold Spring Harb Perspect Biol, 7(1), 123.

12. Martins, P. P., Smyth, H. D., \& Cui, Z. (2019). Strategies to facilitate or block nose-to-brain drug delivery. Int J Pharm, 570, 118635.

13. Bonferoni, M. C., Rossi, S., Sandri, G., Ferrari, F., Gavini, E., Rassu, G., \& Giunchedi, P. (2019). Nanoemulsions for "nose-to-brain" drug delivery. Pharmaceutics, 11(2), 84.

14. Thakur, A., Singh, P. K., Biswal, S. S., Kumar, N., Jha, C. B., Singh, G., ... \& Kumar, R. (2020). Drug delivery through nose: A noninvasive technique for brain targeting. Journal of Reports in Pharmaceutical Sciences, 9(1), 168.

15. Liu, X. F., Fawcett, J. R., Hanson, L. R., \& Frey II, W. H. (2004). The window of opportunity for treatment of focal cerebral ischemic damage with noninvasive intranasal insulin-like growth factor-I in rats. Journal of Stroke and Cerebrovascular Diseases, 13(1), 16-23.

16. Li, Y., Wang, C., Zong, S., Qi, J., Dong, X., Zhao, W., ... \& Chen, Z. (2019). The trigeminal pathway dominates the nose-to-brain transportation of intact polymeric nanoparticles: Evidence from aggregation-caused quenching probes. Journal of biomedical nanotechnology, 15(4), 686-702.

17. Arora, P., Sharma, S., \& Garg, S. (2002). Permeability issues in nasal drug delivery. Drug discovery today, 7(18), 967-975.

18. Schaefer, M. L., Böttger, B., Silver, W. L., \& Finger, T. E. (2002). Trigeminal collaterals in the nasal epithelium and olfactory bulb: a potential route for direct modulation of olfactory information by trigeminal stimuli. Journal of Comparative Neurology, 444(3), 221-226.

19. Lochhead, J. J., \& Thorne, R. G. (2012). Intranasal delivery of biologics to the central nervous system. Advanced drug delivery reviews, 64(7), 614-628.

20. S Hersh, D., S Wadajkar, A., B Roberts, N., G Perez, J., P Connolly, N., Frenkel, V., ... \& J Kim, A. (2016). Evolving drug delivery strategies to overcome the blood brain barrier. Current pharmaceutical design, 22(9), 1177-1193.

21. Agrawal, M., Tripathi, D. K., Saraf, S., Saraf, S., Antimisiaris, S. G., Mourtas, S., ... \& Alexander, A. (2017). Recent advancements in liposomes targeting strategies to cross blood-brain barrier (BBB) for the treatment of Alzheimer's disease. Journal of controlled release, 260, 61-77.

22. Rabanel, J. M., Faivre, J., Paka, G. D., Ramassamy, C., Hildgen, P., \& Banquy, X. (2015). Effect of polymer architecture on curcumin encapsulation and release from PEGylated polymer nanoparticles: Toward a drug delivery nano-platform to the CNS. European Journal of Pharmaceutics and Biopharmaceutics, 96, 409-420.

23. Barbara, R., Belletti, D., Pederzoli, F., Masoni, M., Keller, J., Ballestrazzi, A., ... \& Grabrucker, A. M. (2017). Novel Curcumin loaded nanoparticles engineered for Blood-Brain Barrier crossing and able to disrupt Abeta aggregates. International journal of pharmaceutics, 526(1-2), 413-424.

24. Liu, W., Ye, A., Liu, W., Liu, C., Han, J., \& Singh, H. (2015). Behaviour of liposomes loaded with bovine serum albumin during in vitro digestion. Food chemistry, 175, 16-24..

25. Anoop, V., Cutinho, L. I., Mourya, P., Maxwell, A., Thomas, G., \& Rajput, B. S. (2020). Approaches for encephalic drug delivery using nanomaterials: The current status. Brain Research Bulletin, 155, 184-190.

26. Kong, S. D., Lee, J., Ramachandran, S., Eliceiri, B. P., Shubayev, V. I., Lal, R., \& Jin, S. (2012). Magnetic targeting of nanoparticles across the intact blood-brain barrier. Journal of controlled release, 164(1), 49-57.

27. Cheng, Y., Dai, Q., Morshed, R. A., Fan, X. Wegscheid, M. L., Wainwright, D. A., ... \& Rincón, E. (2014). Blood- brain barrier permeable gold nanoparticles: an efficient delivery platform for enhanced malignant glioma therapy and imaging. Small, 10(24), 5137-5150.

28. Wen, M. M., El-Salamouni, N. S., El-Refaie, W. M., Hazzah, H. A., Ali, M. M., Tosi, G., ... \& Hanafy, A. S. (2017). Nanotechnology-based drug delivery systems for Alzheimer's disease management: Technical, industrial, and clinical challenges. Journal of Controlled Release, 245, 95-107.

29. Shah, B., Khunt, D., Bhatt, H., Misra, M., \& Padh, H. (2015). Application of quality by design approach for intranasal delivery of rivastigmine loaded solid lipid nanoparticles: effect on 
formulation and characterization parameters. European journal of pharmaceutical sciences, 78, 54-66.

30. Tan, X., Kim, G., Lee, D., Oh, J., Kim, M., Piao, C., ... \& Lee, M. (2018). A curcumin-loaded polymeric micelle as a carrier of a microRNA-21 antisense-oligonucleotide for enhanced anti-tumor effects in a glioblastoma animal model. Biomaterials science, 6(2), 407-417.

31. Akel, H., Ismail, R., \& Csóka, I. (2020). Progress and perspectives of brain-targeting lipid-based nanosystems via the nasal route in Alzheimer's disease. European Journal of Pharmaceutics and Biopharmaceutics, 148, 38-53.

32. Siddiqui, R., \& Khan, N. A. (2020). Proposed Intranasal Route for Drug Administration in the Management of Central Nervous System Manifestations of COVID-19. ACS Chemical Neuroscience. 11(11),1523-24.

33. Mungroo, M. R., Khan, N. A., \& Siddiqui, R. (2020). Novel coronavirus: Current understanding of clinical features, diagnosis, pathogenesis, and treatment options. Pathogens, 9(4), 297.

34. Meng, Z., Wang, T., Li, C., Chen, X., Li, L., Qin, X., ... \& Luo, J. (2020). An experimental trial of recombinant human interferon alpha nasal drops to prevent coronavirus disease 2019 in medical staff in an epidemic area. MedRxiv.

35. Lara-Velazquez, M. A., Al-kharboosh, R. M., Norton, E. S., Ramirez-Loera, C., Freeman, W. D., Guerrero-Cazares, H., ... \& Sarabia Estrada, R. (2020). Chitosan-Based Non-viral Gene and Drug Delivery Systems for Brain Cancer. Frontiers in Neurology, 11, 740, 1-10.

36. Jena, L., McErlean, E., \& McCarthy, H. (2020). Delivery across the blood-brain barrier: nanomedicine for glioblastoma multiforme. Drug delivery and translational research, 10(2), 304318.

37. Chung, K., Ullah, I., Kim, N., Lim, J., Shin, J., Lee, S. C., ... \& Lee, S. K. (2020). Intranasal delivery of cancer-targeting doxorubicin-loaded PLGA nanoparticles arrests glioblastoma growth. Journal of drug targeting, 28(6), 617-626.

38. Sabir, F., Ismail, R., \& Csoka, I. (2020). Nose-tobrain delivery of antiglioblastoma drugs embedded into lipid nanocarrier systems: status quo and outlook. Drug discovery today, 25(1), 185-194.

39. Hashizume, R., Ozawa, T., Gryaznov, S. M., Bollen, A. W., Lamborn, K. R., Frey, W. H., \& Deen, D. F. (2008). New therapeutic approach for brain tumors: Intranasal delivery of telomerase inhibitor GRN163. Neuro-oncology, 10(2), 112120.

40. Bruinsmann, F. A., Richter Vaz, G., de Cristo Soares Alves, A., Aguirre, T., Raffin Pohlmann, A., Stanisçuaski Guterres, S., \& Sonvico, F. (2019). Nasal drug delivery of anticancer drugs for the treatment of glioblastoma: preclinical and clinical trials. Molecules, 24(23), 4312.

41. Faria, G. M., Soares, I. D., D’Alincourt Salazar, M., Amorim, M. R., Pessoa, B. L., da Fonseca, C. O., \& Quirico-Santos, T. (2020). Intranasal perillyl alcohol therapy improves survival of patients with recurrent glioblastoma harboring mutant variant for MTHFR rs1801133 polymorphism. BMC cancer, 20, 1-10.

42. Pardridge, W. M. (2020). Blood-Brain Barrier and Delivery of Protein and Gene Therapeutics to Brain. Front Aging Neurosci, 11, 373.

43. Iarkov, A., Barreto, G. E., Grizzell, J. A., \& Echeverria, V. (2020). Strategies for the treatment of Parkinson's disease: Beyond dopamine. Frontiers in Aging Neuroscience, 4(12), 1-20.

44. Yetisgin, A. A., Cetinel, S., Zuvin, M., Kosar, A., \& Kutlu, O. (2020). Therapeutic Nanoparticles and Their Targeted Delivery Applications. Molecules, 25(9), 2193.

45. Islam, S. U., Shehzad, A., Ahmed, M. B., \& Lee, Y. S. (2020). Intranasal Delivery of Nanoformulations: A Potential Way of Treatment for Neurological Disorders. Molecules, 25(8), 1929.

46. Tang S, Wang A, Yan X, Chu L, Yang X, Song Y. (2019). Brain-targeted intranasal delivery of dopamine with borneol and lactoferrin comodified nanoparticles for treating Parkinson's disease. Drug Delivery, 26(1), 700-707.

47. Novak, P., Pimentel Maldonado, D. A., \& Novak, V. (2019). Safety and preliminary efficacy of intranasal insulin for cognitive impairment in Parkinson disease and multiple system atrophy: A double-blinded placebo-controlled pilot study. PloS one, 14(4), e0214364.

48. Santamaria, G., Brandi, E., Vitola, P. L., Grandi, F., Ferrara, G., \& Pischiutta, F. (2020). Intranasal delivery of mesenchymal stem cell secretome repairs the brain of Alzheimer's mice. Cell Death Differ. Doi: https://doi.org/10.1038/s41418-0200592-2.

49. Wang, Z., Xiong, G., Tsang, W. C., Schatzlein, A. G., \& Uchegbu, I. F. (2020). Nose-to-Brain Delivery. J Pharmacol Exp Ther, 370, 593-601.

50. Alexander, A., \& Saraf, S. (2018). Nose-to-brain drug delivery approach: a key to easily accessing the brain for the treatment of Alzheimer's disease. Neural Regen Res, 13(12), 2102-4.

51. Musumeci, T., Bonaccorso, A., \& Puglisi, G. (2019). Epilepsy Disease and Nose-to-Brain Delivery of Polymeric Nanoparticles: An Overview. Pharmaceutics, 11(118), 1-21.

52. Gadhave, D. G., \& Kokare, C. R. (2019). Nanostructured lipid carriers engineered for intranasal delivery of teriflunomide in multiple sclerosis: optimization and in vivo studies. Drug Dev Ind Pharm, 45(5), 839-51. 
53. Rassy, D., Barcena, B., Perez-Osorio, I. N., Espinosa, A., Peon, A. N., \& Terrazas, L. I. (2020). Intranasal Methylprednisolone Effectively Reduces Neuroinflammation in Mice with Experimental Autoimmune Encephalitis. J Neuropathol Exp Neurol, 79(2), 226-37.

54. Khan, R. S., Dine, K., Bauman, B., Lorentson, M., Lin, L., \& Brown, H. (2017). Intranasal Delivery of a Novel Amnion Cell Secretome Prevents Neuronal Damage and Preserves Function in A Mouse Multiple Sclerosis Model. Scientific Reports, 7, 417-68

55. Chen, C., Zhang, M., Wu, Y., Zhou, C., \& Liu, R. (2019). Intranasal Delivering Method in the Treatment of Ischaemic Stroke. In: Chen, J., Wang, J., Wei, L., \& Zhang, J. (Eds) Therapeutic Intranasal Delivery for Stroke and Neurological Disorders. Switzerland: Springer International Publishing.

56. Quintana, D. S., Smerud, K. T., Andreassen, O. A., \& Djupesland, P. G. (2018). Evidences of intranasal oxytocin delivery to the brain: recent advances and future perspectives. Ther Deliv, 9(7), 515-25.

57. Parker, K. J., Oztan, O., Libove, R. A., Mohsin, N., Karhson, D. S., \& Sumiyoshi, R. D. (2019). A randomized placebo-controlled pilot trial shows that intranasal vasopressin improves social deficits in children with autism. Sci Trans Med., 491(11), 7356.

58. Wink, L. K., Reisinger, D. L., Horn, P., Shaffer, R. C., O’Brien, K., \& Schmitt, L. (2020). Brief Report: Intranasal Ketamine in Adolescents and Young Adults with Autism Spectrum Disorder-Initial Results of a Randomized, Controlled, Crossover, Pilot Study. J Autism Dev Disord.

59. Rukmangathen, R., Yallamalli, I. M., \& Yalavarthi, P. R. (2019). Formulation and biopharmaceutical evaluation of risperidoneloaded chitosan nanoparticles for intranasal delivery. Drug Development and Industrial Pharmacy, 45(8), 1342-50. 